\title{
Evolução temporal dos tipos partos em um estado do Nordeste brasileiro
}

\author{
Temporal evolution of childbirth types in a Northeastern Brazilian state \\ Evolución temporal de los tipos de nacimientos en un estado del Noreste de Brasil
}

Recebido: 18/12/2021 | Revisado: 27/12/2021 | Aceito: 10/01/2022| Publicado: 12/01/2022

\author{
Wanderlei Barbosa dos Santos \\ ORCID: https://orcid.org/0000-0001-9813-8857 \\ Universidade Federal de Alagoas, Brasil \\ E-mail: wanderlei.santos@esenfar.ufal.br \\ Amuzza Aylla Pereira dos Santos \\ ORCID: https://orcid.org/0000-0001-6299-7190 \\ Universidade Federal de Alagoas, Brasil \\ E-mail: amuzza.santos@gmail.com \\ Isabel Comassetto \\ ORCID: https://orcid.org/0000-0002-2389-9384 \\ Universidade Federal de Alagoas, Brasil \\ E-mail: isabelcomassetto@gmail.com \\ José Augustinho Mendes Santos \\ ORCID: https://orcid.org/0000-0002-1570-4102 \\ Universidade Federal de Alagoas, Brasil \\ E-mail: augustinhomendes1@gmail.com \\ Deborah Moura Novaes Acioli \\ ORCID: https://orcid.org/0000-0002-3295-8606 \\ Universidade Federal de Alagoas, Brasil \\ E-mail: deborahmnovaes22@gmail.com \\ Mariana Maria Pereira Cintra Farias \\ ORCID: https://orcid.org/0000-0001-5041-5376 \\ Universidade Federal de Alagoas, Brasil \\ E-mail: maripcintra@gmail.com \\ Nathalia Lima Da Silva \\ ORCID: https://orcid.org/0000-0002-5163-7103 \\ Universidade Federal de Alagoas, Brasil \\ E-mail: nathalialimaa17.nl@gmail.com \\ Ana Luiza Souza De Faria Lôbo \\ ORCID: https://orcid.org/0000-0002-8877-0338 \\ Universidade Federal de Alagoas, Brasil \\ E-mail:analuizalobo91@gmail.com
}

\begin{abstract}
Resumo
Objetivo: Analisar a evolução temporal dos tipos partos em um estado do nordeste brasileiro no período entre 2009 a 2018. Métodos: Trata-se de uma pesquisa quantitativa, descritiva, retrospectiva e ecológica, realizada com as Declarações de Nascidos Vivos (DNV) do estado de Alagoas notificados no período entre 2009 e 2018. Foram utilizados dados secundários disponibilizados no Sistema de Informações sobre Nascidos Vivos (SINASC), coletados em outubro de 2020. Resultados: Foram incluídos no estudo 523.005 nascimentos sendo $46,1 \%$ de parto vaginal e 53,9\% de cesariana, quando analisados os dados sociodemográficos maternos houve predomínio da faixa etária de 20 a 29 anos, com escolaridade de 8 a 11 anos e solteiras, apresentaram de 37 a 41 semanas de gestação com mais de 7 consultas realizadas no pré-natal. Conclusão: Evidenciou-se que houve aumento acentuado dos partos cesáreos no período do estudo, que corroboram com a realidade encontrada no país.
\end{abstract}

Palavras-chave: Cesárea; Enfermagem obstétrica; Epidemiologia; Parto normal; Promoção da saúde.

\begin{abstract}
Objective: To analyze the temporal evolution of types of childbirth in a northeastern Brazilian state in the period between 2009 and 2018. Methods: This is a quantitative, descriptive, retrospective, and ecological research, carried out with the Birth Certificates (DNV) of the state from Alagoas reported in the period between 2009 and 2018. Secondary data available in the Information System on Live Births (SINASC), collected in October 2020, were used. Results: 523,005 births were included in the study, $46.1 \%$ of which were vaginal delivery and $53.9 \%$ of cesarean sections, when analyzing the maternal sociodemographic data, there was a predominance of the age group from 20 to 29 years old, with schooling from 8 to 11 years and single, presented from 37 to 41 weeks of gestation with more than 7 consultations performed in the pre- Christmas. Conclusion: It was evident that there was a marked increase in cesarean deliveries during the study period, which corroborates the reality found in the country.
\end{abstract}

Keywords: Cesarean section; Obstetric nursing; Epidemiology; Natural childbirth; Health promotion. 


\section{Resumen}

Objetivo: Analizar la evolución temporal de los tipos de partos en un estado del noreste de Brasil en el período de 2009 a 2018. Métodos: Se trata de una investigación cuantitativa, descriptiva, retrospectiva y ecológica, realizada con las Certificaciones de Nacimiento (DNV) del estado. de Alagoas reportados en el período 2009-2018.Se utilizaron los datos secundarios disponibles en el Sistema de Información de Nacidos Vivos (SINASC), recolectado en octubre de 2020. Resultados: Se incluyeron 523.005 nacimientos en el estudio, $46.1 \%$ de los cuales fueron por parto vaginal. y 53,9\% de las cesáreas, al analizar los datos sociodemográficos maternos, predominó el grupo etario de 20 a 29 años, con escolaridad de 8 a 11 años y soltera, que se presentó de 37 a 41 semanas de gestación con más de 7 consultas realizadas en el pre-navideño. Conclusión: Se evidenció que hubo un marcado aumento de partos por cesárea durante el período de estudio, lo que corrobora la realidad encontrada en el país.

Palabras clave: Cesárea; Enfermería obstétrica; Epidemiología; Parto normal; Promoción de la salud.

\section{Introdução}

O parto ao longo dos tempos, passou de um evento domiciliar, de âmbito familiar, para, a partir do século XX, um evento hospitalar, utilizando-se de métodos e técnicas intervencionistas e do parto operatório. Anteriormente, o parto era considerado um evento fisiológico e nesse momento havia o protagonismo feminino no processo de parir. Com o advento e desenvolvimento da medicina, o parto tornou-se predominantemente institucionalizado e a mulher nesse novo contexto era coadjuvante do ato de parir, já que necessitava de intervenção médica e amparo medicamentoso e cirúrgico (Silvaet al., 2019; Damasceno et al., 2018).

É importante ressaltar que o modelo de assistência ao parto no Brasil, apesar da existência de programas de apoio a diminuição das intervenções na assistência ao parto e os movimentos femininos contra a falta de protagonismo feminino no parto, ainda apresenta um modelo obstétrico que se caracteriza pelas elevadas taxas de cesarianas e como consequência elevado número de mortalidade materna e perinatal (Rocha \& Ferreira 2020).

Ainda é percebido que o número de partos operatórios vem aumentando de forma significativa e que se faz necessário alertar, que quando as indicações são desnecessárias aumenta o potencial de risco iatrogênico. Sendo imprescindível revelar que mesmo utilizando melhorias nos métodos e tecnologias, assim como o avanço com o uso da cesariana, ao invés de diminuir a morbimortalidade materna e neonatal aumentaram os índices de prematuridade e morbimortalidade materna e neonatal (Rego \& Matão, 2016).

Na tentativa de melhorar a realidade de assistência ao parto, o Ministério da saúde (MS) designou o Programa de Humanização no Pré-Natal e Nascimento (PHPN), esse programa tem o objetivo de unificar e propor a vinculação entre os serviços de pré-natal e parto, garantindo o acesso a assistência à gestante no pré-natal e maternidades. Com a finalidade de reforçar essa assistência foi instituída a Rede cegonha com a proposta de melhorar a saúde da mulher no ciclo gravídico puerperal e reduzir as taxas de mortalidade materna e neonatal (Gonçalves et al., 2017).

Nesse contexto, o processo de humanização do parto vem com o intuito de fortalecer a proposta dos programas ministeriais, assumindo um papel de respeitar o parto como sendo um evento fisiológico, assim como o protagonismo feminino, através de boas práticas obstétricas alicerçadas em evidências científicas favorecendo a desconstrução de uma assistência intervencionista e fria que se utiliza do uso abusivo de técnicas e métodos invasivos desnecessários e nocivos a fisiologia do parto. Essa adequação tem como propósito reduzir mortes evitáveis de mulheres e recém-nascidos e garantir uma assistência de qualidade a essas mulheres (Melo et al., 2020).

Desta forma, este estudo torna-se relevante pelo conhecimento da evolução dos partos ocorridos em um Estado situado na região nordeste do Brasil, no período dos últimos 10 anos, e que irá contribuir para as equipes multidisciplinares traçarem estratégias e melhorias na qualidade da assistência prestada à mulher durante a evolução da sua gestação. Nesse sentido o estudo traz como questão norteadora: Qual a evolução temporal dos tipos partos ocorridos em um estado do nordeste no período compreendido entre 2009 a 2018? Com isto, o objetivo deste estudo é analisar a evolução temporal dos tipos partos em um Estado do nordeste brasileiro no período entre 2009 a 2018. 


\section{Metodologia}

Trata-se de um estudo quantitativo, com abordagem descritiva, retrospectiva e ecológica, realizado a partir das Declarações de Nascidos Vivos (DNV) do estado de Alagoas, e dos nascimentos notificados no período de 2009 a 2018. Para realização do estudo foram utilizados os dados secundários disponibilizados via internet do Sistema de Informações sobre Nascidos Vivos (SINASC), os quais foram coletados no mês de outubro de 2020.

Em relação às variáveis de interesse para este estudo, considerou-se como variável independente o tipo de parto, sendo o mesmo classificado em vaginal e cesáreo. Já em as variáveis dependentes, foram divididas em: variáveis sociodemográficas maternas (idade, escolaridade e estado civil), variáveis obstétricas (duração da gestação, tipo de gravidez e número de consultas de pré-natal) e variáveis do recém-nascido (sexo, raça/cor, Apgar no $1^{\circ}$ e $5^{\circ}$, peso ao nascer e presença de malformações congênitas).

O processamento dos dados foi feito através do TAB para Windows-TabWin (DATASUS). Trata-se de um aplicativo que permite tabular informações de diferentes tipos em um mesmo ambiente. Após, os dados foram transferidos para uma planilha no Microsoft Excel e em seguida foram realizadas e analisadas a frequência absoluta e relativa dos dados.

Em relação aos aspectos éticos, por se tratar de um estudo onde os dados foram coletados em uma plataforma de domínio público, essa pesquisa não necessitou ser submetida ao Comitê de Ética em Pesquisa.

\section{Resultados}

No período de 2009 a 2018 foram notificados 524.058 nascimentos no estado de Alagoas. Destes, 241.230 foram nascimentos de parto vaginal, enquanto 281.775 foram de parto cesáreo. Vale destacar que foram excluídos deste estudo 1.053 nascimentos, visto que a informação tipo do parto foi ignorada no momento do preenchimento da DNV. Com a exclusão, foram analisados 523.005 nascimentos de acordo com o tipo de parto, sendo 46,1\% nascimentos de parto vaginal e 53,9\% de parto cesáreo.

Ao analisar o Gráfico 1, é possível observar o percentual de acordo com o tipo de parto, por ano de nascimento. 
Gráfico 1: Evolução do percentual do tipo de parto. Alagoas-BR, 2009-2018

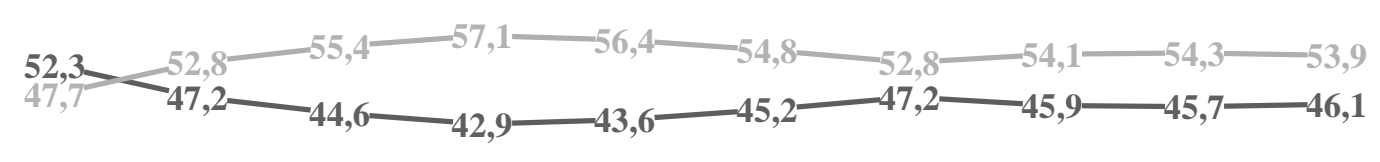

$\begin{array}{cccccccccc}2009 & 2010 & 2011 & 2012 & 2013 & 2014 & 2015 & 2016 & 2017 & 2018 \\ & - & -\mathrm{PV} & - & -\mathrm{PC}\end{array}$

Legenda: PV- parto vaginal; PC- parto cesáreo. Fonte: Sistema de Informação sobre Nascidos Vivos (SINASC).

Em relação às características sociodemográficas maternas, observa-se que, ao analisar os nascimentos de forma geral, $51,1 \%$ são filhos de mães com idade entre 20 a 29 anos (44,5\%), que possui escolaridade de 8 a 11 anos de estudo e solteiras $(39,3 \%)$. Quando analisado por tipo de parto, a idade materna e escolaridade são semelhantes em ambos os grupos, porém observa-se que com relação ao estado civil, 37,1\% das mulheres casadas tiveram seus filhos de parto cesáreo, enquanto 43,5\% das mulheres solteiras tiveram seus filhos por parto vaginal (Tabela 1). 
Tabela 1: Características sociodemográficas maternas gerais $(n=523.005)$ e de acordo com o tipo de parto (vaginal $n=241.230$ e cesáreo ( $\mathrm{n}=281.775)$. Alagoas-BR, 2009-2018.

\begin{tabular}{|c|c|c|c|c|c|c|}
\hline \multirow[t]{2}{*}{ VARIÁVEIS } & \multicolumn{2}{|c|}{ GERAL } & \multicolumn{2}{|c|}{ PARTO VAGINAL } & \multicolumn{2}{|c|}{ PARTO CESÁRIO } \\
\hline & $\mathbf{N}$ & $\%$ & $\mathbf{N}$ & $\%$ & $\mathbf{N}$ & $\%$ \\
\hline \multicolumn{7}{|l|}{ IDADE } \\
\hline 10 a 19 anos & 132.176 & $25,3 \%$ & 77.172 & $32,0 \%$ & 55.004 & $19,5 \%$ \\
\hline 20 a 29 anos & 267.015 & $51,1 \%$ & 121.075 & $50,2 \%$ & 145.940 & $51,8 \%$ \\
\hline 30 a 39 anos & 113.960 & $21,8 \%$ & 39.128 & $16,2 \%$ & 74.832 & $26,6 \%$ \\
\hline 40 a 49 anos & 9.854 & $1,9 \%$ & 3.855 & $1,6 \%$ & 5.999 & $2,1 \%$ \\
\hline \multicolumn{7}{|l|}{ ESCOLARIDADE } \\
\hline Nenhuma & 14.509 & $2,8 \%$ & 9.876 & $4,1 \%$ & 4.633 & $1,6 \%$ \\
\hline 1 a 3 anos & 47.619 & $9,1 \%$ & 28.794 & $11,9 \%$ & 18.825 & $6,7 \%$ \\
\hline 4 a 7 & 163.207 & $31,2 \%$ & 88.934 & $36,9 \%$ & 74.273 & $26,4 \%$ \\
\hline 8 a 11 & 232.616 & $44,5 \%$ & 97.776 & $40,5 \%$ & 134.840 & $47,9 \%$ \\
\hline 12 ou mais & 55.645 & $10,6 \%$ & 10.560 & $4,4 \%$ & 45.085 & $16,0 \%$ \\
\hline Ignorado & 9.409 & $1,8 \%$ & 5.290 & $2,2 \%$ & 4.119 & $1,5 \%$ \\
\hline \multicolumn{7}{|l|}{ ESTADO CIVIL } \\
\hline Solteira & 205.341 & $39,3 \%$ & 104.906 & $43,5 \%$ & 100.435 & $35,6 \%$ \\
\hline Casada & 158.531 & $30,3 \%$ & 54.099 & $22,4 \%$ & 104.432 & $37,1 \%$ \\
\hline Viúva & 1.014 & $0,2 \%$ & 437 & $0,2 \%$ & 577 & $0,2 \%$ \\
\hline Separada & 2.261 & $0,4 \%$ & 724 & $0,3 \%$ & 1.537 & $0,5 \%$ \\
\hline União Consensual & 148.286 & $28,4 \%$ & 76.501 & $31,7 \%$ & 71.785 & $25,5 \%$ \\
\hline Ignorado & 7.572 & $1,4 \%$ & 4.563 & $1,9 \%$ & 3.009 & $1,1 \%$ \\
\hline
\end{tabular}

Fonte: Sistema de Informação sobre Nascidos Vivos (SINASC).

Em relação às características obstétricas, em ambos os grupos, tiveram a duração da gestação entre 37 e 41 semanas e gravidez do tipo feto único. Pode-se observar que com relação a gravidez duplas ou mais, 2,6\% nasceram de parto cesáreo e $1,2 \%$ de parto vaginal. Já em relação ao número de consultas de pré-natal, a maioria realizou 7 ou mais consultas (Tabela 2). 
Tabela 2: Características obstétricas gerais $(\mathrm{n}=523.005)$ de acordo com o tipo de parto (vaginal n=241.230 e cesáreo n=281.775). Alagoas-BR, 2009-2018.

\begin{tabular}{|c|c|c|c|c|c|c|}
\hline \multirow[t]{2}{*}{ VARIÁVEIS } & \multicolumn{2}{|l|}{ GERAL } & \multicolumn{2}{|c|}{ PARTO VAGINAL } & \multicolumn{2}{|c|}{ PARTO CESÁREO } \\
\hline & $\mathbf{N}$ & $\%$ & $\mathbf{N}$ & $\%$ & $\mathbf{N}$ & $\%$ \\
\hline \multicolumn{7}{|c|}{ DURAÇÃO DA GESTAÇÃO } \\
\hline$\leq 36$ semanas & 50.136 & $9,6 \%$ & 24.300 & $10,1 \%$ & 25.836 & $9,2 \%$ \\
\hline 37 a 41 semanas & 417.366 & $79,8 \%$ & 188.356 & $78,1 \%$ & 229.010 & $81,3 \%$ \\
\hline$\geq 42 \mathrm{~s}$ semanas & 19.499 & $3,7 \%$ & 9.441 & $3,9 \%$ & 10.058 & $3,6 \%$ \\
\hline Ignorado & 36.004 & $6,9 \%$ & 19.133 & $7,9 \%$ & 16.871 & $6,0 \%$ \\
\hline \multicolumn{7}{|l|}{ TIPO DE GRAVIDEZ } \\
\hline Única & 513.660 & $98,2 \%$ & 238.596 & $98,9 \%$ & 275.064 & $97,6 \%$ \\
\hline Dupla & 8.469 & $1,6 \%$ & 2.267 & $0,9 \%$ & 6.202 & $2,2 \%$ \\
\hline Tripla ou mais & 183 & $0,0 \%$ & 73 & $0,03 \%$ & 110 & $0,04 \%$ \\
\hline Ignorado & 693 & $0,1 \%$ & 294 & $0,1 \%$ & 399 & $0,1 \%$ \\
\hline \multicolumn{7}{|c|}{ CONSULTAS DE PRÉ-NATAL } \\
\hline Nenhuma a 3 consultas & 63.368 & $12,1 \%$ & 38.947 & $16,1 \%$ & 24.421 & $8,7 \%$ \\
\hline 4 a 6 consultas & 192.979 & $36,9 \%$ & 97.660 & $40,5 \%$ & 95.319 & $33,8 \%$ \\
\hline 7 ou mais & 262.251 & $50,1 \%$ & 102.653 & $42,6 \%$ & 159.598 & $56,6 \%$ \\
\hline Ignorado & 4.407 & $0,8 \%$ & 1.970 & $0,8 \%$ & 2.437 & $0,9 \%$ \\
\hline
\end{tabular}

Fonte: Sistema de Informação sobre Nascidos Vivos (SINASC).

\section{Discussão}

O procedimento do parto cesáreo que teve a princípio o objetivo de salvar a vida das mulheres que tinham algum impedimento ou distocia para o parto vaginal, acompanhou a evolução humana e passou por aprimoramentos e atualmente possui ampla aceitação nos partos eletivos. Mesmo com os riscos inerentes do procedimento, as mulheres optam pelo parto cesáreo e alguns fatores estariam relacionados a essa escolha, como os medos e tabus do parto vaginal, uma visão equivocada da realização do procedimento cirúrgico e a própria estética dessa mulher (Agrawal \& Sahoo, 2019; Rosenberg \& Trevathan, 2018).

Neste tipo de procedimento são observados alguns riscos inerentes do próprio processo cirúrgico, como a infecção pósparto, hemorragia, permanência na unidade de terapia intensiva, traumas e mortalidade materna, ficando evidente o agravante que as mulheres se expõem a essa modalidade de parto, principalmente nos casos que não existe indicação (Mascarello, Horta \& Silveira, 2017). Além dos riscos supracitados, outro dado que pode ser evidenciado se refere aos custos para a realização do procedimento, quando comparados os custos dos partos vaginais. Observa-se um valor menor dos partos vaginais, no entanto, para realizar a cesariana é necessária uma gestão financeira e recursos humanos para a realização da cirurgia, no Brasil em média os custos para realizar a cesariana é de $R \$ 1.113,70$ e o parto vaginal $R \$ 808,16$, isto mostra a economia que poderia ser gerada para o sistema público de saúde, tendo em vista que são partos com cobertura da gestão pública (Entringer, Pinto \& Gomes, 2019; Entringer et al. 2018).

$\mathrm{Na}$ verificação da evolução dos partos, torna-se importante mencionar as características sociodemográficas das mulheres, nesta pesquisa a faixa etária de 20 a 29 anos foi mais evidenciada nos partos vaginais e cesáreos com percentuais similares, quando comparado com os dados de um estudo realizado em um hospital público na região norte do país, evidenciou que os partos foram predominantes nesta mesma faixa etária, corroborando com os dados desta pesquisa, possivelmente essa fase adulta jovem das mulheres apresenta o melhor período para a gravidez, pois as mulheres estão com suas funções anatômicas e fisiológicas preparadas para gestação (Barbosa et al., 2017). 
Outras características como escolaridade e estado civil devem ser mencionados, as mulheres desta pesquisa apresentavam em média de 8 a 11 anos de estudo e em sua maioria as mulheres são solteiras, que apresentaram maior número de partos vaginais, e para os partos cesáreos os percentuais foram maiores nas mulheres casadas. A escolaridade de ensino médio completo foi observada em outro estudo realizado no estado do Ceará, essa variável contribui para o esclarecimento de medidas de promoção da saúde que são realizadas durante o pré-natal, mostrando a finalidade dos dois tipos de partos e permitindo uma garantia da autonomia para a mulher ter o direito de escolha da via de parto (Silva et al., 2019; Andrade et al., 2018).

Em relação à situação conjugal, alguns estudos referem que as mulheres apresentam união estável ou são casadas nos partos realizados em unidades privadas ou particulares, ressalta-se que a presença masculina tem interferência positiva para o binômio, pois possibilita a mulher uma segurança maior em uma fase frágil da sua vida. A situação conjugal de solteira também poderia estar relacionada com alguns fatores de situação econômica e de vulnerabilidades que poderiam contribuir para situações de risco para a mãe e o filho (Andrade et al., 2018; Kuzma et al., 2016).

A idade gestacional (IG) também foi caracterizada neste estudo com taxas do parto cesáreo superiores aos vaginais em todas as IGs. Com base em estudos da OMS, foi proposto que a taxa ideal para os partos operatórios seria de $10 \%$ a $15 \%$, entretanto, o Brasil ainda mantém taxas superiores a 70\%, principalmente quando se refere ao setor privado (Rossetto et al., 2020).

Neste estudo foi observado que quanto maior o número de consultas pré-natal, maior as taxas de cesariana. Diante do exposto, evidenciou-se que as mulheres que fizeram um acompanhamento completo no pré-natal, conforme o preconizado no Ministério da saúde, tiveram como desfecho o parto cesáreo, enquanto as que evoluíram para o parto vaginal fizeram menos de 6 consultas de pré-natal (Fonsecal et al., 2019). É importante apontar que a evolução do parto pode ser consequência de condições predisponentes à cesariana, como hipertensão, pré-eclâmpsia e diabetes gestacional, o que determinam uma maior frequência nas consultas de pré-natal. É importante ressaltar que o Ministério da Saúde recomenda que para um pré-natal adequado, deve ser iniciado até o quarto mês de gestação, sendo composto por, no mínimo, seis consultas de acompanhamento em gestação de risco habitual (Silva et al., 2020).

Quando analisada a cobertura do pré-natal foi observado uma concentração de mulheres pretas ou pardas na região norte e nordeste do país, esse grupo historicamente apresenta uma maior vulnerabilidade o que pode refletir no seu processo gravídicopuerperal, estas podem apresentar risco de pré-natal inadequado, dificuldades de acesso às maternidades e possíveis complicações no parto. As mulheres pardas ou pretas apresentaram maior incidência de partos vaginais, no entanto, quando utilizados recursos como anestesia durante a episiotomia, elas receberam menos medicamentos quando comparadas com as mulheres brancas, além de ficarem mais expostas às violências obstétricas durante a assistência (Leal et al., 2017; Diniz et al., 2016).

Desta forma, foi observado que vários fatores contribuem para o desfecho dos partos ocorridos no país, e como é importante a realização do pré-natal para a promoção e prevenção de agravos que possam contribuir na qualidade de vida da mulher no seu período gravídico-puerperal.

\section{Conclusão}

A realidade encontrada no estudo demostra que a evolução dos tipos de partos mostrou-se semelhante à encontrada no país, com maior quantitativo dos partos cesáreos no período de 2009 a 2018. Ressalta-se que as mulheres que foram mais propensas a cesariana estava na fase adulta jovem, expondo estas mulheres aos riscos inerentes do procedimento, como o desfecho da mortalidade materna. Além disso, os impactos para a saúde pública são relevantes nos custos causados pela cirurgia, pós cirurgias e as possíveis complicações destas. 
Os dados apresentados nesta pesquisa mostram a relevância deste tema no estado e possibilita o suporte para que novos estudos sejam realizados, permitindo a criação de estratégias que permitam as mulheres conhecerem as vias de parto e seus benefícios, sendo trabalhados no planejamento familiar e no pré-natal, para contribuir com o empoderamento e autonomia da mulher, além da diminuição das taxas de cesáreas no país.

\section{Referências}

Agrawal, R. \& Sahoo, G. (2019). Evolution of Cesarean Section. Journal of Medical Science And clinical Research, 07(04): 193-201.

Andrade, S. G., Vasconcelos, Y. A., Carneiro, A. R. S., Severiano, A. R. G., Terceiro, A. J. M. D. \& Silva, T. B., et al. (2018). Perfil sociodemográfico, epidemiológico e obstétrico de parturientes em um hospital e maternidade de Sobral, Ceará. Rev Pre Infec e Saúde, 4(7283): 1-13.

Barbosa, E. M., Oliveira, A. S. S., Galiza, D. D. F., Barros, V. L., Aguiar, V. F. \& Marques, M. B. (2017). Perfil sociodemográfico e obstétrico de parturientes de um hospital público. Rev Rene, 18(2):227-33.

Damasceno, V. C., Caliman, L. P., Mezzonato Machado, N. C., Gonçalves, A. B. C. \& Mendes de Miranda, L. C. (2018). Taxa de cesariana nas primigestas atendidas numa maternidade pública com assistência humanizada no município de Juiz de Fora - MG. HU ver, $43(2)$ : 121 - 126.

Diniz, C. S. G., Batista, L. E., Kalckmann, S., Schlithz, A. O. C., Queiroz, M. R. \& Carvalho, P. C. A. (2016). Desigualdades sociodemográficas e na assistência à maternidade entre puérperas no Sudeste do Brasil segundo cor da pele: dados do inquérito nacional Nascer no Brasil (2011-2012). Saude soc. 25(3):561-572.

Entringer, A. P., Gomes, M. A. S. M., Costa, A. C. C. \& Pinto, M. (2018). Impacto orçamentário do parto vaginal espontâneo e da cesariana eletiva sem indicação clínica no Brasil. Rev Panam Salud Publica, 42(e116): 1-7.

Entringer, A. P., Pinto, M. F. T. \& Gomes, M. A. S. M. (2019). Análise de custos da atenção hospitalar ao parto vaginal e à cesariana eletiva para gestantes de risco habitual no Sistema Único de Saúde. Ciênc. Saúde Colet. 24(4): 1527-1536.

Fonsecal, R., Nogueira, K., Alves, F. \& Lima, L. (2019). Caracterização epidemiológica dos partos em uma cidade no interior do ceará: estudo ecológico com base em um sistema de informação. Encontro De Extensão, Docência e Iniciação Científica (EEDIC). 6: 1-6.

Gonçalves, M. F., Teixeira, É. M. B., Silva, M. A. S., Corsi, N. M., Ferrari, R. A. P. \& Pelloso S. M. et al. (2017). Pré-natal: preparo para o parto na atenção primária à saúde no sul do Brasil. Rev. gaúch. enferm., 38(3): 1-8.

Kuzma, G. S. P., Reiter, M. G. R., Carrocini, M. M. S., Morelli, S. R. \& Venzon, P. P. (2016). Perfil de puérperas assistidas em alojamento conjunto: Estudo comparativo entre os serviços público e privado. Rev AMRIGS. 60(2): 87-91.

Leal, M. C., Gama, S. G. N., Pereira, A. P. E., Pacheco, V. E., Carmo, C. N. \& Santos, R. V. (2017) A cor da dor: iniquidades raciais na atenção pré-natal e ao parto no Brasil. Cad Saúde Pública. 33(Suppl 1): 1-17.

Mascarello, K. C., Horta, B. L. \& Silveira, M. F. (2017). Maternal complications and cesarean section without indication: systematic review and meta-analysis. Rev. Saúde Públ, 51(105): 1-12.

Melo, A. A., Diaz, C. M. G., Zamberlan, C., Antunes, B., Marques, C. T., \& Silveira, G. B., et al. (2020). Perfil da assistência ao parto em maternidade de risco usual: tipo de parto e intervenções. Research, Society and Development., 9(2): 1-10.

Rego, M. B. C. \& Matão, M. E. L. (2016). Análise dos partos vaginais e cesarianas no município de Goiânia-goiás: antes e após a rede cegonha. Revista da Universidade Vale do Rio Verde, Três Corações, 14(2): 83-92.

Rocha, N. F. F. \& Ferreira, J. (2020). A escolha da via de parto e a autonomia das mulheres no Brasil: uma revisão integrativa. Saúde debate, 44(125): 556-568.

Rosenberg, K. R. \& Trevathan, W. R. (2018). Evolutionary perspectives on cesarean section. Evol Med Public Health, $2018(1)$ : 67-81.

Rossetto, M., Schmalfuss, J., Bedin, K., Pinheiro, A. \& Batista, J. (2020). Fatores associados à cesariana eletiva em mulheres atendidas em um hospital referência do oeste catarinense. Rev. Enferm. UFSM, 10(e54): 1-17.

Silva, E. V., Costa, M. A. A., Almeida, K. C., Araujo, L. M. B. \& Amâncio, N. F. G. (2020). Relação do tipo de parto com o perfil epidemiológico da assistência pré-natal e perinatal em um município de Minas Gerais. Rev. Bras. Saude Mater. Infant. 20(1): 241-247.

Silva, M.C. R. G., Silva, L. S. R., Sousa, J. O., Frota, M. C. Q. A., Carneiro, J. K. R. \& Oliveira, M. A. S. (2019). Perfil epidemiológico-obstétrico e sociodemográfico de gestantes atendidas em um Centro de Saúde da Família. Revista Saúde e Desenvolvimento. 13(14): 100-111.

Silva, F., Nucci, M., Nakano, A. R. \& Teixeira, L. (2019). "Parto ideal”: medicalização e construção de uma roteirização da assistência ao parto hospitalar no Brasil em meados do século XX1. Saúde e Sociedade, 28(3): 171-184. 